\title{
Different airway inflammatory responses in asthmatic and healthy humans exposed to diesel
}

\author{
N. Stenfors*, C. Nordenhäll*, S.S. Salvi", I. Mudway ", M. Söderberg*, A. Blomberg*, R. Helleday*, \\ J-O. Levin ${ }^{+}$, S.T. Holgate", F.J. Kelly", A.J. Frew", T. Sandström*
}

Different airway inflammatory responses in asthmatic and healthy humans exposed to diesel. N. Stenfors, C. Nordenhäll, S.S. Salvi, I. Mudway, M. Söderberg, A. Blomberg, R. Helleday, J-O. Levin, S. T. Holgate, F.J. Kelly, A.J. Frew, T. Sandström. (C) ERS Journals Ltd 2004. ABSTRACT: Particulate matter (PM) pollution adversely affects the airways, with asthmatic subjects thought to be especially sensitive. The authors hypothesised that exposure to diesel exhaust (DE), a major source of PM, would induce airway neutrophilia in healthy subjects, and that either these responses would be exaggerated in subjects with mild allergic asthma, or DE would exacerbate pre-existent allergic airways.

Healthy and mild asthmatic subjects were exposed for $2 \mathrm{~h}$ to ambient levels of DE (particles with a 50\% cut-off aerodynamic diameter of $10 \mu \mathrm{m}(\mathrm{PM} 10) 108 \mu \mathrm{g} \cdot \mathrm{m}^{-3}$ ) and lung function and airway inflammation were assessed.

Both groups showed an increase in airway resistance of similar magnitude after DE exposure. Healthy subjects developed airway inflammation $6 \mathrm{~h}$ after DE exposure, with airways neutrophilia and lymphocytosis together with an increase in interleukin-8 (IL8) protein in lavage fluid, increased IL-8 messenger ribonucleic acid expression in the bronchial mucosa and upregulation of the endothelial adhesion molecules. In asthmatic subjects, DE exposure did not induce a neutrophilic response or exacerbate their preexisting eosinophilic airway inflammation. Epithelial staining for the cytokine IL-10 was increased after DE in the asthmatic group.

Differential effects on the airways of healthy subjects and asthmatics of particles with a 50\% cut-off aerodynamic diameter of $10 \mu \mathrm{m}$ at concentrations below current World Health Organisation air quality standards have been observed in this study. Further work is required to elucidate the significance of these differential responses.

Eur Respir J 2004; 23: 82-86.
*Dept of Respiratory Medicine and Allergy, University Hospital, Umeå, Sweden, ${ }^{*}$ Respiratory Cell and Molecular Biology Research Division, Southampton General Hospital, Southampton, UK, Cardiovascular Research, the Rayne's Institute, St Thomas Hospital, London, UK and ${ }^{+}$National Institute for Working Life, Umeå, Sweden.

Correspondence: T. Sandström, Dept of Respiratory Medicine and Allergy, University Hospital, SE-901 85, Umeå, Sweden.

Fax: 4690141369

E-mail: Thomas.sandstrom@lung.umu.se

Keywords: Airway inflammation, asthma, cytokines, diesel, pollution

Received: January 142003

Accepted: July 282003

This study was supported by the Health Effects Institute, Cambridge, MA, USA, the National Asthma Campaign, UK, the Dept of Health, UK, the Swedish Heart Lung Foundation, Umeå University and the Wellcome Trust.
Numerous epidemiological studies have demonstrated clear associations between increased ambient particulate matter (PM) concentrations and indices of pulmonary and cardiac morbidity and mortality within the general population [1]. These adverse effects appear to be magnified in populations with pre-existing respiratory disease, such as asthma [2]. During PM pollution episodes, asthmatic subjects demonstrate increased respiratory symptoms, bronchoconstriction, medication use, bronchial hyperreactivity and emergency care visits $[2,3]$. The mechanism underlying this difference remains unclear.

Exposure to particles with a $50 \%$ cut-off aerodynamic diameter of $10 \mu \mathrm{m}$ (PM10) at concentrations above the USA National Air Quality Standards of $150 \mu \mathrm{g} \cdot \mathrm{m}^{-3}$ (24 h average) occurs frequently in many cities throughout the world, due to a combination of vehicle traffic and industrial processes. The transport sector, especially diesel-powered vehicles, is a major source of urban PM pollution. Diesel exhaust (DE) is a complex mixture containing carbonaceous particles, oxides of nitrogen, carbon monoxide, aldehydes and other volatile organic carbon species. In addition, DE particles may act as vectors for the delivery to the lung of toxic materials, including heavy metal ions, hydrocarbons and allergens $[4,5]$.

The authors have previously shown airway inflammatory responses in healthy volunteers exposed to DE at a PM10 concentration of $300 \mu \mathrm{g} \cdot \mathrm{m}^{-3}[6,7]$. In the present study the airway responses of mildly asthmatic (MA) and healthy control (HC) adults to a lower, and environmentally more relevant, concentration of DE (PM10 $108 \mu \mathrm{g} \cdot \mathrm{m}^{-3}$ for $2 \mathrm{~h}$ ) with intermittent exercise as a surrogate for environmental PM pollution, have been assessed. The authors hypothesised that short-term exposure to ambient levels of DE would induce neutrophilic airway inflammation in healthy subjects, and that either these responses would be exaggerated in subjects with mild allergic asthma, or diesel would exacerbate preexistent allergic airways.

\section{Materials and methods}

\section{Study subjects}

A total of 25 healthy non-atopic subjects (nine females; mean age 24 yrs (range 19-42)) and 15 subjects with mild asthma [8], using only inhaled $\beta_{2}$-agonists on demand (five females; mean age 30 yrs (range 22-52)) were recruited. The asthmatics reacted to at least one airborne allergen skin test and showed airways hyperresponsiveness to methacholine (geometric mean provocative dose causing a $20 \%$ fall in forced expiratory volume in one second $(\mathrm{FEV} 1) 1.6 \mathrm{mg} \cdot \mathrm{mL}^{-1}$; range $\left.0.3-6 \mathrm{mg} \cdot \mathrm{mL}^{-1}\right)$. All subjects were free of airway infection for 6 weeks prior to and throughout the study. Subjects were never-smokers and had normal baseline spirometry. No anti-inflammatory or other drugs were permitted during the study, which was conducted outside the pollen season. All 
participants gave their informed consent and the local Ethics Committee approved the study.

\section{Study design}

All subjects were exposed to filtered air and DE (PM10 $100 \mu \mathrm{g} \cdot \mathrm{m}^{-3}, 0.7$ parts per million (ppm) nitrogen dioxide $\left(\mathrm{NO}_{2}\right)$ ) during $2 \mathrm{~h}$ on two separate occasions. The exposures were carried out at least 3 weeks apart and in a randomised single-blind manner. During exposures, the volunteers alternated moderate exercise and rest. Lung function responses were assessed before, during and immediately after exposures. Bronchoscopy was done $6 \mathrm{~h}$ after each exposure to obtain mucosal biopsies and airway lavages.

\section{Methods}

DE was generated by an idling Volvo diesel engine (Volvo, Gothenburg, Sweden). Over $90 \%$ of the exhaust was discarded; the remainder being diluted with air and fed into the exposure chamber [6, 9]. Exposures were conducted in random order; subjects and investigators were blind to exposure sequence, but engineering staff supervising the chamber were aware of exposure details. During exposures, volunteers alternately exercised on a bicycle ergometer (ventilation/perfusion ratio= $15-20 \mathrm{~L} \cdot \mathrm{min}^{-1} \cdot \mathrm{m}^{-2}$ ) and rested for $15 \mathrm{~min}$ periods.

Lung function responses were assessed using a computerised whole body plethysmograph (System 2800, Sensor Medics Corp., CA, USA). Forced vital capacity (FVC) and FEV1 were measured before and immediately after exposures. Specific airway resistance (sRaw) was measured immediately before exposure, $1 \mathrm{~h}$ into the exposure, and immediately after the exposure.

Bronchoscopy was performed $6 \mathrm{~h}$ after ceasing exposure as previously described [6]. Prior to bronchoscopy the asthmatic subjects inhaled $0.2 \mathrm{mg}$ salbutamol dry powder. Bronchial biopsies were taken from proximal cristae. Bronchial wash ((BW) $2 \times 20 \mathrm{~mL})$ and bronchoalveolar lavage $((\mathrm{BAL}) 3 \times 60 \mathrm{~mL})$ were carried out in the contralateral lung. BW and BAL were analysed for differential cell counts, albumin, total protein, IL-6, IL-8, granulocyte macrophage colony stimulating factor (GM-CSF), methyl-histamine, myeloperoxidase (MPO) and eosinophil cationic protein (ECP).

Mucosal biopsies were processed into glycolmethacrylate resin, cut and stained using monoclonal antibodies directed against specific cellular markers, vascular endothelial adhesion molecules (P-selectin, E-selectin, vascular adhesion molecule (VCAM)-1 and intracellular adhesion molecule (ICAM)-1), cytokines and transcription factors (GM-CSF, growth-related oncogene alpha (Gro- $\alpha$ ), IL-6, IL-8, IL-10, nuclear factor $(\mathrm{NF})-\kappa \mathrm{B}$, regulated on activation, normal T-cell expressed and secreted (RANTES) and tumour necrosis factor- $\alpha$ (TNF- $\alpha$ )) $[6,7]$. Stained inflammatory cells were counted in the epithelium and submucosa excluding glands, blood vessels and muscle. The counts were expressed as cells $\cdot \mathrm{mm}^{-1}$ in the epithelium and cells $\cdot \mathrm{mm}^{-2}$ in the submucosa. The length of the epithelium and the area of the submucosa were calculated using a computerassisted image analyser (Colour Vision Software, Improvision System, Birmingham, UK). Endothelial adhesion molecules in the vessels were quantified by expressing the proportion of vessels staining with monoclonal antibodies as a percentage of the total number of blood vessel stained with the endothelial marker EN4 in adjacent $2 \mu \mathrm{m}$ sections. Total ribonucleic acid was extracted from bronchial biopsies and reverse transcriptase polymerase chain reaction enzyme-linked immunosorbent assay EMG electromyogram was performed to quantify relative changes in IL- $1 \beta$, IL-5, IL- $8, \mathrm{TNF}-\alpha$, interferon gamma (IFN- $\gamma$ ) and cytokine messenger ribonucleic acid
(mRNA) gene transcripts [10]. Before comparing between treatments (exposure to air and exposure to DE), the levels of cytokine transcripts were normalised to adenine phosphoribosyl transferase (APRT) and expressed as a percentage (level of cytokine products/level of APRT product $\times 100$ ).

\section{Analysis}

Within-group changes were analysed using Wilcoxon's paired rank test. Between group differences were compared by Mann-Whitney U-test. FEV1 and FVC responses were compared by repeated measures two way analysis of variance. Post-hoc analysis was performed using the Student-NewmanKuels test. The HC and MA responses were compared using an unpaired t-test. sRaw responses were tested with a general linear method, repeated measurements with sRaw measurements during exposures as dependent variables. The sRaw results during exposures were considered dependent variables. The values were compared within groups, air versus diesel, as well as between groups, healthy versus asthmatic subjects. $\mathrm{P}$-values $<0.05$ were considered significant.

\section{Results}

The steady state PM10 and gaseous concentrations in the exposure chamber were: PM10 $108 \mathrm{ug} \cdot \mathrm{m}^{-3}$ (94-124) (mean (range)), carbon monoxide $1.7 \mathrm{ppm}$ (0.6-2.5), nitric oxide $0.6 \mathrm{ppm}(0.5-0.6), \mathrm{NO}_{2} 0.2 \mathrm{ppm}(0.1-0.3)$, hydrocarbons $1.4 \mathrm{ppm}$ (1.3-1.8) and formaldehyde $43.5 \mathrm{ug} \cdot \mathrm{m}^{-3}$ [33-53]. Differential mobility particle sizer measurements of DE yielded geometric mean electrical mobility equivalent diameters for particle number, particle surface and particle volume of $0.073,0.12$ and $0.28 \mu \mathrm{m}$, respectively.

\section{Lung function}

No significant differences in pre-air FEV1 or FVC were noted between the groups, and DE exposure did not affect these parameters in either group. The MA group had significantly greater $\mathrm{s}$ Raw before air exposures compared to $\mathrm{HC}$ subjects (mean $\pm \mathrm{SD}$ ) $0.547 \pm 0.202 \mathrm{kPa}$ in MA versus $0.408 \pm$ 0.122 in $\mathrm{HC} ; \mathrm{p}<0.01$. In healthy subjects, exposure to $\mathrm{DE}$ induced a significant increase in $\mathrm{s} R$ aw of $4.1 \%$, compared to air exposures, $\mathrm{p}<0.01$, whereas the increase in the asthmatic group was $6.5 \%, \mathrm{p}<0.01$. There was no statistical difference in the magnitude of response between groups.

\section{Healthy control group}

In the $\mathrm{HC}$ group, exposure to DE induced an increase in the proportion of $\mathrm{BW}$ neutrophils $(\mathrm{p}<0.05)$. The staining of the bronchial biopsies showed a trend toward a decrease in mucosal neutrophils after DE (43.4 (15.6-94.6) (median (interquartile range)) cells $\cdot \mathrm{mm}^{-2}$ post-air versus 24.6 (16.8-57.1) cells $\cdot \mathrm{mm}^{-2}$ post-DE, though this did not reach statistical significance (fig. 1). A significant increase in both the relative $(\mathrm{p}<0.05)$ and total lymphocyte numbers was seen in the BAL samples after DE $\left(1.5(1.2-1.8) \times 10^{4}\right.$ cells $\cdot \mathrm{mL}^{-1}$ post-air versus $2.0(1.3-2.6)$ post-DE; $\mathrm{p}<0.05)$ and there was a parallel decrease in CD3+ lymphocytes in the bronchial epithelium (3.0 (1.5$6.1)$ cells $\cdot \mathrm{mm}^{-1}$ post-air versus $1.7(0.4-3.1)$ post-DE; $\left.\mathrm{p}<0.05\right)$.

The neutrophil recruitment into the proximal airways was accompanied by significant increases in the protein concentrations of the pro-inflammatory cytokines IL-6 and IL-8 in BW (table 1). This was associated with increased IL- 8 mRNA gene transcripts in the proximal bronchial tissue (51.0 (13.4 $65.1) \%$ APRT post-air versus 65.7 (30.7-84.5) \% APRT post-DE; 


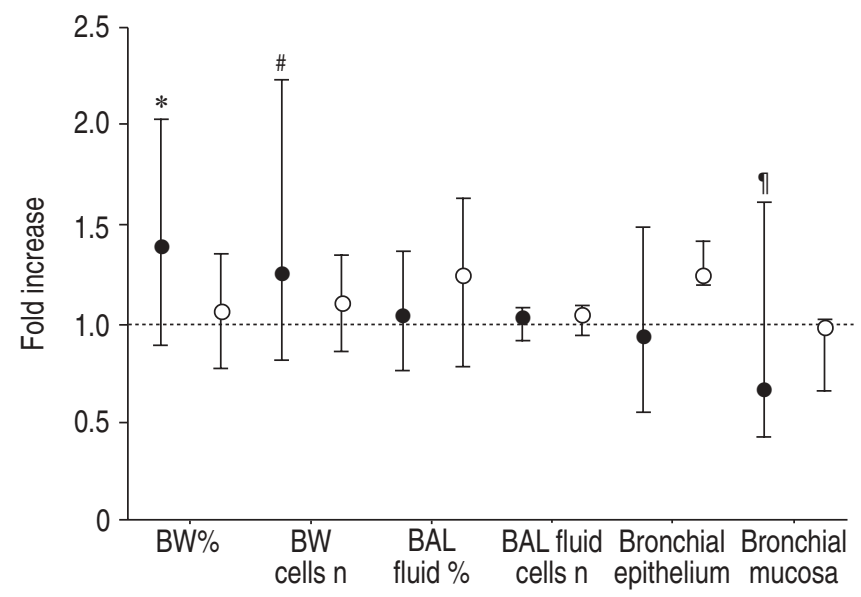

Fig. 1.-Change in neutrophil numbers after exposure to diesel exhaust versus filtered air in asthmatic ( $)$ and healthy subjects $(\bigcirc)$. Data are given for airway compartments sampled with complementary techniques; bronchial wash (BW), percentage neutrophils of all cells and total neutrophil number, Bronchoalveolar lavage (BAL), percentage neutrophils of all cells and total neutrophil number, neutrophils in the bronchial epithelium and neutrophils in the bronchial submucosa. Error bars show interquartile range. ${ }^{\#}: \mathrm{p}=0.07 ;{ }^{\uparrow}: \mathrm{p}=0.14{ }^{*}: \mathrm{p}<0.05$.

$\mathrm{p}<0.05)$ and increased expression of P-selectin $(\mathrm{p}<0.005)$ and VCAM-1 $(\mathrm{p}<0.05)$ in the proximal bronchial mucosa after $\mathrm{DE}$ (table 2). There were no DE-induced changes in BW or BAL concentrations of MPO, GM-CSF, total protein or albumin.

\section{Mild asthmatic group}

Consistent with their asthmatic status there were a number of differences in the samples obtained from the MA group after air exposure, compared with the corresponding samples from the $\mathrm{HC}$ group (table 3 ). These included higher numbers of eosinophils in BW $(\mathrm{p}<0.001)$ and in the bronchial mucosa $(\mathrm{p}<0.001)$, with an associated elevation in BW ECP levels $(\mathrm{p}<0.05)$. Asthmatic subjects had significantly greater BW mast cell numbers $(\mathrm{p}<0.05)$ and methyl-histamine concentrations $(\mathrm{p}=0.001)$, but lower baseline BAL lymphocyte counts $(\mathrm{p}<0.05)$, and fewer CD3+ lymphocytes in bronchial epithelium $(\mathrm{p}<0.01)$. Diesel exposure did not affect ECP or methylhistamine concentrations in BW or BAL in either group. The MA group had higher levels of IL-8 mRNA $(\mathrm{p}<0.05)$, and altered expression of ICAM-1 and VCAM-1 (table 2).

After DE exposure, the MA subjects showed a small decrease in BW eosinophils $(0.9(0.2-1.55) \%$ post-air versus 0.4 $(0.0-0.85) \%$ post-DE, $\mathrm{p}<0.05)$ but no change in eosinophil numbers in BAL or in bronchial mucosa. DE exposure did not affect neutrophil or lymphocyte numbers in BW or BAL in MA, nor did it affect VCAM-1 or P-selectin expression in bronchial biopsies (table 2 ).

Several inflammatory and regulatory cytokines were assessed for their possible contribution to the process of airways inflammation, including IL-10 (figs. 2 and 3). There was a higher baseline expression of TNF- $\alpha$ in the MA group (median (interquartile range): $0.18(0.0-0.70) \%$ in MA versus 0.0 $(0.0-0.0)$ in $\mathrm{HC} ; \mathrm{p}<0.05)$ and a lower density of IL-10 expression $(0.22(0.14-0.68) \%$ in MA versus $1.29(0.45-2.51)$ in $\mathrm{HC}$; $\mathrm{p}=0.001$ ) (fig. 2a). Following DE exposure, IL-10 staining increased markedly in the MA group $(0.22(0.14-0.68) \%$ post-air versus 0.99 (0.63-1.08) post-DE; $\mathrm{p}<0.01)$ (fig. 2b). There was no significant change in epithelial staining for IL-6, IL-8, GM-CSF, Gro- $\alpha$, RANTES, TNF- $\alpha$ or NF- $\kappa$ B in either group following exposure to DE.

\section{Discussion}

In this study healthy and mild asthmatic subjects were exposed to DE at PM10 concentrations below USA National Air Quality Standards. Both groups showed an increase in sRaw of similar magnitude after DE. As the authors hypothesised, DE induced airway inflammation in healthy subjects. This consisted of a mild BW neutrophilia and BAL lymphocytosis, together with an increase in IL- 6 and IL-8 protein in

Table 1. - Concentration of interleukin (IL)-8 and IL-6 protein in bronchial wash of healthy control subjects and mild asthmatics exposed to diesel exhaust (DE) fumes and air

\begin{tabular}{|c|c|c|c|c|c|}
\hline & \multicolumn{2}{|c|}{$\mathrm{IL}-8 \mathrm{pg} \cdot \mathrm{mL}^{-1}$} & \multicolumn{3}{|c|}{$\mathrm{IL}-6 \mathrm{pg} \cdot \mathrm{mL}^{-1}$} \\
\hline & Air & $\mathrm{DE}$ & Air & & $\mathrm{DE}$ \\
\hline Healthy controls & \multirow[t]{2}{*}{ (1.0) } & $54.0(30.0-74.8)$ & $3.3(1.8-5.4)$ & \multirow{2}{*}{$\mathrm{p}<0.05$} & $5.1(1.8-9.3)$ \\
\hline Mild asthmatics & & $44.0(27.3-51.3)$ & $2.0(1.6-6.0)$ & & $3.8(2.3-10.9)$ \\
\hline
\end{tabular}

Data are presented as median (interquartile range). NS: nonsignificant.

Table 2.-Percentage of total EN4 staining vessels expressing the named adhesion molecule on the vascular endothelium of healthy and mild asthmatic subjects exposed to diesel exhaust (DE) fumes

\begin{tabular}{|c|c|c|c|c|}
\hline & E-selectin \% & P-selectin \% & VCAM-1 \% & ICAM-1 \% \\
\hline \multicolumn{5}{|c|}{ Healthy controls } \\
\hline Air & $20.2(11.3-27.0)$ & $52.5(50.0-64.9)$ & $4.8(2.3-10.1)$ & $64.1(58.5-71.6)$ \\
\hline $\mathrm{DE}$ & $20.3(15.3-30.0)$ & $65.4(58.4-75.5)$ & $8.8(4.6-12.3)$ & $66.7(59.4-80.0)$ \\
\hline p-value & NS & $\mathrm{p}<0.01$ & $\mathrm{p}<0.05$ & NS \\
\hline \multicolumn{5}{|c|}{ Mild asthmatics } \\
\hline Air & $16.0(11.8-21.9)$ & $50.7(36.8-58.1)$ & $12.5(9.1-17.8)^{*}$ & $56.2(45.9-63.4)$ \\
\hline $\mathrm{DE}$ & $16.7(11.7-22.4)$ & $54.5(47.3-61.2)$ & $8.3(2.7-11.1)$ & $52.7(41.7-59.6)$ \\
\hline $\mathrm{p}$-value & NS & NS & NS & NS \\
\hline
\end{tabular}

Data are presented median (interquartile range). Baseline post-air differences between groups are indicated with *: p $<0.05$. VCAM-1: vascular adhesion molecule; ICAM: intracellular adhesion molecule; NS: nonsignificant. 
Table 3. - Differences between healthy control and mild asthmatic groups after air exposure

\begin{tabular}{|c|c|c|c|c|}
\hline Parameter & Healthy & Asthmatics & Units & p-value \\
\hline BW eosinophils & $0.0(0.00-0.35)$ & $0.79(0.28-2.00)$ & $\times 10^{3}$ cells $\cdot \mathrm{mL}^{-1}$ & $<0.001$ \\
\hline BBx eosinophils & $0.0(0.0-1.8)$ & $4.9(2.4-10.3)$ & cells $\cdot \mathrm{mm}^{-2}$ & $<0.001$ \\
\hline BW ECP & $0.94(0.89-1.30)$ & $1.7(1.0-2.04)$ & $\mu \mathrm{g} \cdot \mathrm{L}^{-1}$ & $<0.05$ \\
\hline IL-8 mRNA & $51.0(13.4-65.1)$ & $71.0(56.9-90.1)$ & $\%$ APRT & $<0.05$ \\
\hline BAL lymphocytes & $1.5(1.2-1.8)$ & $0.8(0.6-1.5)$ & $\times 10^{4}$ cells $\cdot \mathrm{mL}^{-1}$ & $<0.05$ \\
\hline $\mathrm{BBx}$ CD3+ cells & $3.0(1.5-6.1)$ & $1.7(0.5-3.3)$ & cells $\cdot \mathrm{mm}^{-1}$ & $<0.01$ \\
\hline BW mast cells & $0.04(0.00-0.09)$ & $0.09(0.04-0.22)$ & $\times 10^{3}$ cells $\cdot \mathrm{mL}^{-1}$ & $<0.05$ \\
\hline BW methyl histamine & $55(0-68)$ & $90(70-100)$ & $\mathrm{ng} \cdot \mathrm{L}^{-1}$ & $=0.001$ \\
\hline
\end{tabular}

BW: bronchial wash; BBx: bronchial biopsy; IL-8: interleukin 8; APRT: adenine phosphoribosyl transferase; BAL: bronchoalveolar lavage.
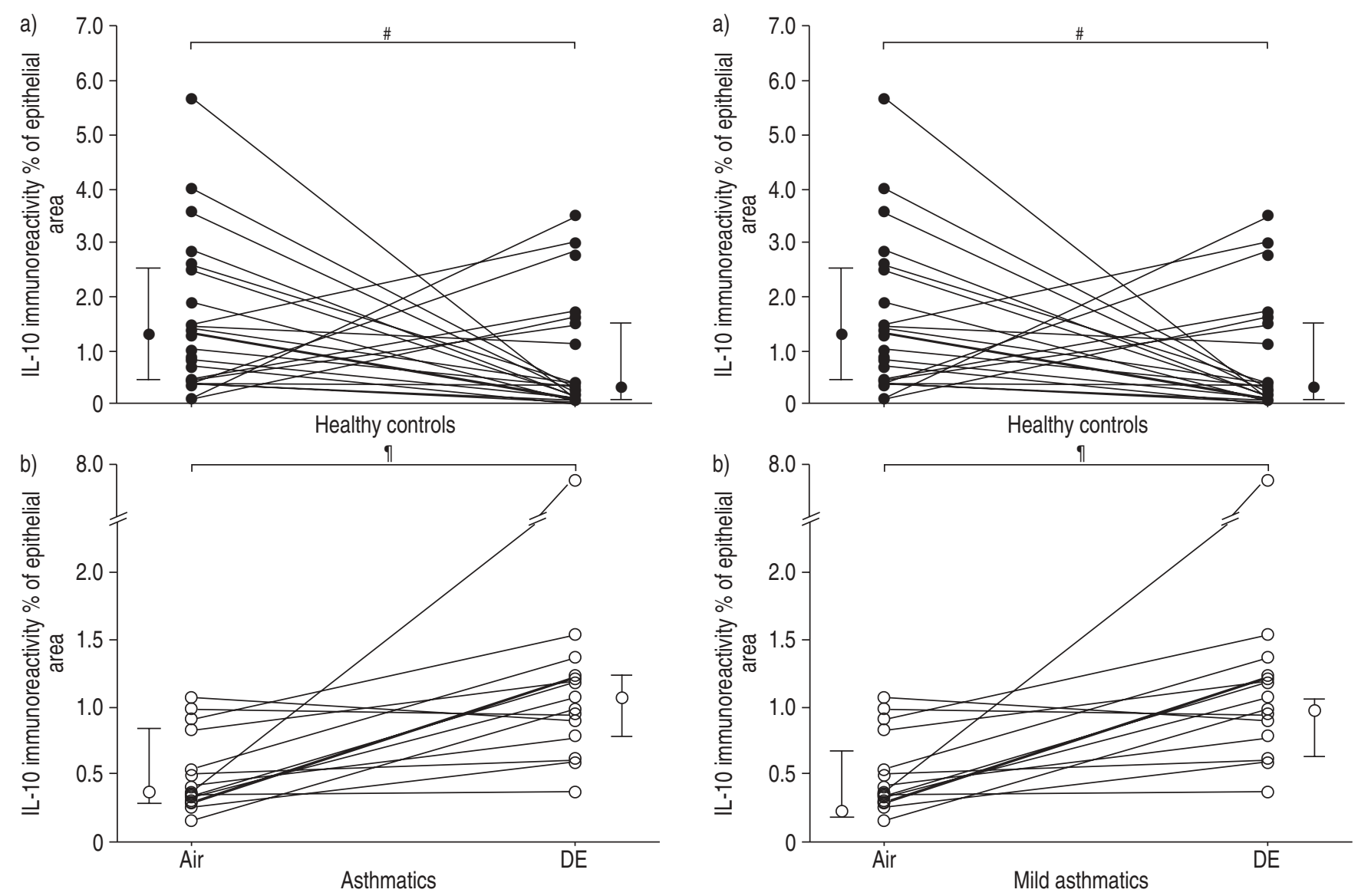

Fig. 2. - Change in bronchial epithelial interleukin (IL)-10 expression after diesel exhaust (DE) versus filtered air exposure for a) healthy subjects and b) asthmatics. Data are presented as per cent of the total measured bronchial epithelial area. Separate points to right and left of main data points show median \pm interquartile range. ${ }^{\text {: }}$ nonsignificant difference; ${ }^{\bullet}: \mathrm{p}=0.002$.

lavage fluid, increased IL-8 mRNA expression in the bronchial mucosa and upregulation of the endothelial adhesion molecules P-selectin and VCAM-1. The MA group showed eosinophilic airways inflammation at baseline but, contrary to the authors hypotheses, exposure to DE neither exacerbated this nor induced an acute neutrophil influx. However, the MA group showed a significant increase in epithelial staining for IL-10 after DE exposure, contrasting to a reduction in IL-10 staining in the HC subjects.

Consistent with previous studies using higher DE concentrations $[6,11,12]$, DE exposure induced a neutrophilic airway inflammation in the $\mathrm{HC}$ subjects. A decrease in submucosal neutrophils was simultaneously observed in the bronchial

Fig. 3. - Change in bronchial epithelial interleukin (IL)-10 expression after diesel exhaust (DE) exposure versus filtered air exposure for a) healthy subjects and b) mild asthmatics. Data are presented as per cent of the total measured bronchial epithelial area. Separate points to right and left of main data points show median \pm interquartile range. ${ }^{\#}$ : nonsignificant difference; ${ }^{\text {: }} \mathrm{p}=0.002$.

biopsies, suggesting movement of cells from the airway wall into the airway lumen. Recruitment of neutrophils and T-cells is usually dependent upon the upregulation of vascular endothelial adhesion molecules. The DE-induced upregulation of VCAM1 and P-selectin suggests early inflammatory cell recruitment from the blood vessels into the bronchial mucosa. In contrast to a previous study by the authors using higher concentrations of DE [6], no increase in mucosal neutrophils was observed. This indicates that challenge with a lower concentration of DE induces either no mucosal neutrophilia or a slower onset of airway inflammation. Importantly, all the inflammatory effects were detected mainly in the proximal airways, suggesting that this is the major site of impact of DE. The 
only response seen in the distal airways was an increase in BAL lymphocytes.

In the MA subjects, the authors had expected to see either a neutrophilic response or an exacerbation of pre-existent eosinophilic airways inflammation, but in fact neither occurred. The enhanced asthmatic sensitivity, such as bronchoconstriction and airway symptoms, observed in epidemiological settings does not seem to be explained by magnified acute airway inflammatory responses in this subpopulation. There are several possible explanations for this. First, the time-point selected may have been too early to detect the cellular response of MA subjects to DE. The $6 \mathrm{~h}$ post-DE time-point was chosen to correspond with the peak cellular response in studies of allergen challenge [13]. It is possible that a cellular response to DE might have been seen in the MA group at a later time-point, even though a response to DE was seen at $6 \mathrm{~h}$ in the HC group. Asthmatic subjects might have a slower response to $\mathrm{DE}$ than $\mathrm{HC}$ subjects, due to endogenous antiinflammatory and immunomodulatory mechanisms that might be operative in the inflamed asthmatic airway and which might dampen or delay the acute inflammatory response to DE/PM challenge. Prostaglandin $\mathrm{E}_{2}$, IL-10 and IFN- $\gamma$ have been identified as such potential inhibitory mechanisms [14]. IL-10 inhibits the synthesis of many proinflammatory cytokines and chemokines, such as IL-6 and IL-8, and baseline levels are low in asthma [14-17]. The IL-10 response seen after DE exposure in the MA group may thus represent a downregulatory response and hence explain the lack of neutrophil, IL-6 or IL-8 response in the MA group. Secondly, although the cells, cytokines and chemokines that are conventionally considered to be important in allergic airway inflammation were measured, there may be other aspects of airway response that are more relevant to DE exposure. The adverse airway effects of PM in asthmatic patients might arise through heightened nonspecific bronchial hyperresponsiveness or enhanced reactivity to allergen challenge [18], indices that were not assessed in this study. Thirdly, although equivalent increases in sRaw were observed in the $\mathrm{HC}$ and MA subjects after diesel exposure, this might have more clinical impact on the MA subjects who started with a higher baseline airways resistance.

One of the clearest differences between MA and $\mathrm{HC}$ in their response to DE was the IL-10 response. Although it has been suggested that IL-10 might be an antiasthmatic cytokine [14], IL-10 also has some properties that could promote allergic airways inflammation. For example, IL-10 potentiates IgE production by B-cells [19] and is a growth factor for mast cells [20]. Induction of IL-10 by DE could thus contribute to skewing the immune system in the airways mucosa towards enhancement of asthmatic airways inflammation.

In conclusion, differential, albeit small, effects on the airways of healthy subjects and asthmatics by particles with a $50 \%$ cut-off aerodynamic diameter of $10 \mu \mathrm{m}$ concentrations below current World Health Organisation air quality standards have been observed. This is consistent with epidemiological observations that small changes in particulate matter concentrations may have significant health effects. This data suggests a direct effect of diesel exhaust on interleukin-8 production, with upregulation of endothelial adhesion molecules and neutrophil recruitment in healthy human airways, whereas mild asthmatics respond with an induction of epithelial interleukin-10. Further work is required to elucidate the significance of these differential responses.

Acknowledgements. The authors wish to thank J. Cameron, J. Pourazar, A-B. Lundström, L. Skedebrant, M-C. Ledin, G. Lindén, B. Rudell and H. Bertilsson for their expert technical assistance.

\section{References}

1. Health effects of outdoor air pollution. Committee of the Environmental and Occupational Health Assembly of the American Thoracic Society. Am J Respir Crit Care Med 1996; 153: 3-50.

2. Boezen M, Schouten J, Rijcken B, et al. Peak expiratory flow variability, bronchial responsiveness, and susceptibility to ambient air pollution in adults. Am J Respir Crit Care Med 1998; 158: 1848-1854.

3. Schwartz J, Slater D, Larson TV, Pierson WE, Koenig JQ. Particulate air pollution and hospital emergency room visits for asthma in Seattle. Am Rev Respir Dis 1993; 147: 826-831.

4. Schroeder WH, Dobson M, Kane DM, Johnson ND. Toxic trace elements associated with airborne particulate matter: a review. JAPCA 1987; 37: 1267-1285.

5. Knox RB, Suphioglu C, Taylor P, et al. Major grass pollen allergen Lol $\mathrm{p} 1$ binds to diesel exhaust particles: implications for asthma and air pollution. Clin Exp Allergy 1997; 27: 246-251.

6. Salvi S, Blomberg A, Rudell B, Kelly F, et al. Acute inflammatory responses in the airways and peripheral blood after short-term exposure to diesel exhaust in healthy human volunteers. Am J Respir Crit Care Med 1999; 159: 702-709.

7. Salvi SS, Nordenhall C, Blomberg A, et al. Acute exposure to diesel exhaust increases IL-8 and GRO-alpha production in healthy human airways. Am J Respir Crit Care Med 2000; 161: $550-557$.

8. National Institute of Health, National Heart LaBI. Global Strategy for Asthma Management and Prevention NHLBI/ WHO Workshop. 95-3659. 1-1-1995.

9. Rudell B, Sandstrom T, Hammarstrom U, Ledin ML, Horstedt P, Stjernberg N. Evaluation of an exposure setup for studying effects of diesel exhaust in humans. Int Arch Occup Environ Health 1994; 66: 77-83.

10. Salvi S, Semper A, Blomberg A, et al. Interleukin-5 Production by Human Airway Epithelial Cells. Am J Respir Cell Mol Biol 1999; 20: 984-991.

11. Nightingale JA, Maggs R, Cullinan P, et al. Airway inflammation after controlled exposure to diesel exhaust particulates. Am J Respir Crit Care Med 2000; 162: 161-166.

12. Rudell B, Sandström T, Stjernberg N, Kolmodin-Hedman B. Controlled diesel exhaust exposure in an exposure chamber: pulmonary effects invetigated with bronchoalveolor lavage. J Aerosol Sci 1990; 21: Suppl. 1, 411-414.

13. Montefort S, Gratziou C, Goulding D, et al. Bronchial biopsy evidence for leukocyte infiltration and upregulation of leukocyte-endothelial cell adhesion molecules 6 hours after local allergen challenge of sensitized asthmatic airways. J Clin Invest 1994; 93: 1411-1421.

14. Barnes PJ, Lim S. Inhibitory cytokines in asthma. Mol Med Today 1998; 4: 452-458.

15. Borish L, Aarons A, Rumbyrt J, Cvietusa P, Negri J, Wenzel S. Interleukin-10 regulation in normal subjects and patients with asthma. J Allergy Clin Immunol 1996; 97: 1288-1296.

16. Sanchez-Guerrero I, Vegara RP, Herrero N, Garcia-Alonso AM, Luna A, Alvarez MR. Cytokine serum profiles in allergic and non-allergic asthma. Increased production of IL10 by non-allergic asthmatic patients. Allergol Immunopathol Madr 1997; 25: 98-103.

17. Takanashi S, Hasegawa Y, Kanehira Y, et al. Interleukin-10 level in sputum is reduced in bronchial asthma, COPD and in smokers. Eur Respir J 1999; 14: 309-314.

18. Diaz-Sanchez D. The role of diesel exhaust particles and their associated polyaromatic hydrocarbons in the induction of allergic airway disease. Allergy 1997; 52: Suppl. 38, 52-56.

19. Jeannin P, Lecoanet S, Delneste Y, Gauchat JF, Bonnefoy JY. IgE versus IgG4 production can be differentially regulated by IL-10. J Immunol 1998; 160: 3555-3561.

20. Thompson-Snipes L, Dhar V, Bond MW, Mosmann TR, Moore KW, Rennick DM. Interleukin 10: a novel stimulatory factor for mast cells and their progenitors. J Exp Med 1991; 173: 507-510. 